\title{
Endovascular Therapy or Alteplase in Patients with Comorbidities: Insights from UNMASK EVT
}

\author{
Aravind Ganesh (1D, Nima Kashani, Johanna M. Ospel, Alexis T. Wilson, \\ Mona M. Foss, Gustavo Saposnik, Mohammed A. Al-Mekhlafi, Mayank Goyal, \\ Bijoy K. Menon, Michael D. Hill 1
}

\begin{abstract}
Objective: Decisions to treat large-vessel occlusion with endovascular therapy (EVT) or intravenous alteplase depend on how physicians weigh benefits against risks when considering patients' comorbidities. We explored EVT/alteplase decision-making by stroke experts in the setting of comorbidity/disability. Methods: In an international multi-disciplinary survey, experts chose treatment approaches under current resources and under assumed ideal conditions for 10 of 22 randomly assigned case scenarios. Five included comorbidities (cancer, cardiac/respiratory/renal disease, mild cognitive impairment [MCI], physical dependence). We examined scenario/respondent characteristics associated with EVT/alteplase decisions using multivariable logistic regressions. Results: Among 607 physicians (38 countries), EVT was chosen less often in comorbidity-related scenarios (79.6\% under current resources, $82.7 \%$ assuming ideal conditions) versus six "level-1A" scenarios for which EVT/alteplase was clearly indicated by current guidelines $(91.1 \%$ and $95.1 \%$, respectively, odds ratio [OR] [current resources]: $0.38,95 \%$ confidence interval $0.31-0.47)$. However, EVT was chosen more often in comorbidity-related scenarios compared to all other 17 scenarios $(79.6 \%$ versus $74.4 \%$ under current resources, OR: 1.34, 1.17-1.54). Responses favoring alteplase for comorbidity-related scenarios (e.g. 75.0\% under current resources) were comparable to level-1A scenarios $(72.2 \%)$ and higher than all others $(60.4 \%)$. No comorbidity independently diminished EVT odds when considering all scenarios. MCI and dependence carried higher alteplase odds; cancer and cardiac/respiratory/renal disease had lower odds. Being older/female carried lower EVT odds. Relevant respondent characteristics included performing more EVT cases/year (higher EVT-, lower alteplase odds), practicing in East Asia (higher EVT odds), and in interventional neuroradiology (lower alteplase odds vs neurology). Conclusion: Moderate-to-severe comorbidities did not consistently deter experts from EVT, suggesting equipoise about withholding EVT based on comorbidities. However, alteplase was often foregone when respondents chose EVT. Differences in decision-making by patient age/sex merit further study.
\end{abstract}

RÉSUMÉ : Traitement endovasculaire ou administration d'altéplase chez des patients présentant des comorbidités : un aperçu de l'étude Unmask EVT. Objectif : La décision de traiter l'occlusion d'un vaisseau sanguin important au moyen d'un traitement endovasculaire (TEV) ou d'altéplase administré par intraveineuse dépend de la façon dont un médecin, en tenant compte des comorbidités de ses patients, pèse les avantages et les risques associés à ces traitements. Nous avons donc cherché ici à mieux comprendre le processus de prise de décision des spécialistes de l'AVC en lien avec un TEV et l'altéplase. Méthodes : En réponse à 10 scénarios cliniques sur 22 attribués au hasard dans le cadre d'un sondage multidisciplinaire international, divers spécialistes ont été amenés à opter pour des approches de traitement sur la base des ressources actuelles et de conditions de pratique idéales. Sur ces 10 scénarios cliniques, 5 incluaient des patients atteints de comorbidités (cancer ; maladie cardiaque, respiratoire et rénale ; trouble cognitif léger ou TCL ; dépendance physique). Pour notre part, c'est au moyen de régressions logistiques multivariées que nous nous sommes penchés sur les scénarios et les caractéristiques des répondants et sur leurs décisions en faveur d'un TEV et de l'altéplase. Résultats : Parmi les 607 médecins spécialistes issus de 38 pays ayant répondu au sondage, un TEV a été choisi moins fréquemment dans les cas de scénarios présentant une comorbidité (79,6\% sur la base des ressources actuelles et $82,7 \%$ en fonction de conditions de pratique idéales) en comparaison avec six scénarios de niveau « $1 \mathrm{~A} »$ pour lesquels un TEV ou l'altéplase étaient clairement indiqués dans les lignes directrices actuelles $(91,1 \%$ et $95,1 \%$ respectivement ; rapport de cotes [ressources actuelles] : 0,38; IC $95 \%$ 0,31-0,47). Cela dit, un TEV a été choisi plus fréquemment dans le cas de scénarios présentant une comorbidité si on les compare à tous les 17 autres scénarios $(79,6 \%$ contre $74,4 \%$ sur la base des ressources actuelles ; rapport de cotes : 1,34;1,17 - 1,54). D'autre part, les réponses favorisant l'altéplase dans le cas de scénarios présentant une comorbidité (à savoir 75,0\% en tenant compte des ressources actuelles) se sont révélées comparables à celles données à des scénarios de niveau «1A » $(72,2 \%)$ et plus nombreuses que toutes les autres $(60,4 \%)$. Fait à noter, aucune comorbidité n'a par ailleurs diminué de façon indépendante la probabilité d'opter pour un TEV si l'on tient compte de tous les scénarios. Une plus grande probabilité d'administrer de l'altéplase a été associée à des TCL et à des problèmes de dépendance ; à l'inverse, le cancer ainsi que des maladies

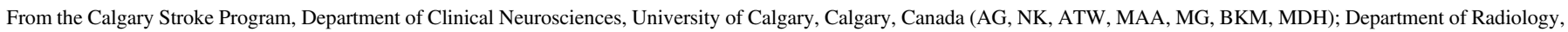

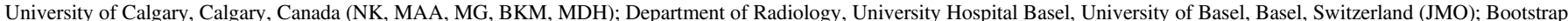

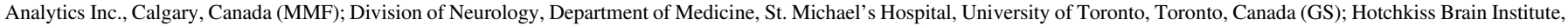
Calgary, Canada (MAA, MG, BKM, MDH); and Departments of Community Health Sciences and Medicine, University of Calgary, Calgary, Canada (BKM, MDH)

Received April 22, 2020. Final Revisions Submitted June 11, 2020. Date of Acceptance July 12, 2020.

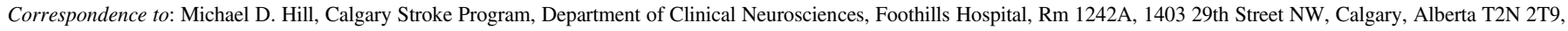
Canada. Email: michael.hill@ucalgary.ca 
cardiaques, respiratoires et rénales ont été associés à une faible probabilité d'administrer de l'altéplase. Le fait d'être plus âgé et de sexe féminin a été associé à une plus faible probabilité de bénéficier d'un TEV. Parmi les caractéristiques de nos répondants jugées pertinentes, mentionnons le fait de réaliser davantage de TEV par année, un plus grand nombre de TEV étant associé à une faible probabilité d'administrer de l'altéplase ; de pratiquer la médecine en Asie de l'Est (probabilité plus élevée de recourir à un TEV); et d'être spécialisé en neuroradiologie interventionnelle (plus faible probabilité d'administrer de l'altéplase en comparaison avec la neurologie). Conclusion : Des comorbidités modérées à sévères n'ont pas systématiquement dissuadé ces spécialistes de recourir à un TEV, ce qui suggère une forme d'incertitude absolue (equipoise) quant au refus de privilégier un TEV en raison de comorbidités. Les répondants ont toutefois souvent renoncé à l'altéplase après avoir opté pour un TEV. En somme, des différences en fonction de l'âge et du sexe des patients quant à la prise de décisions méritent des études plus approfondies.

Keywords: All cerebrovascular disease/stroke, Comorbidity, Endovascular therapy, Premorbid disability, Thrombolysis

doi:10.1017/cjn.2020.158

Can J Neurol Sci. 2021; 48: 77-86

\section{INTRODUCTION}

Endovascular therapy (EVT) is highly efficacious for acute ischemic stroke due to large-vessel occlusion, ${ }^{1}$ up to 24 -hours post-onset in select cases. ${ }^{2,3}$ Thrombolysis with alteplase is efficacious in ischemic stroke within 4.5 hours of onset ${ }^{4}$ and in selected patients in later time windows. ${ }^{5,6}$ However, randomized evidence is lacking in patients with multiple comorbidities or pre-stroke disability, who have generally been excluded from stroke trials. ${ }^{7}$ For instance, most EVT/alteplase trials excluded patients with premorbid modified Rankin Scale (mRS) score $\geq 2$, although they may constitute up to one-third of all patients with ischemic stroke. ${ }^{8}$ This approach is not based on any mechanistic hypothesis about reduced benefit, but simply reflects the fact that such patients cannot contribute to typical dichotomy-based definitions of favorable outcome (e.g. mRS $0-1$ or $0-2){ }^{9}$ Nevertheless, comorbidities and premorbid disability will commonly cluster together (as comorbid illness commonly causes disability) and are often cited as a reason for excluding patients from acute therapies. ${ }^{10}$

The reticence to treat patients with high prestroke disability/ comorbidity is partly due to their poorer prognosis, with registry data reporting high mortality and complication rates. ${ }^{8,11}$ Although post-stroke mortality has improved over the past two decades, comorbidity burden remains a strong prognostic factor. ${ }^{12}$ Comorbidities like hypertension and diabetes are associated with poorer outcomes after thrombolysis or EVT, particularly among older patients. ${ }^{13-16}$ However, stroke commonly occurs in patients with multiple comorbidities, and in acute stroke settings, the prognosis of these conditions may be elusive. ${ }^{17}$ Decisions to treat with EVT/alteplase will partly depend on how physicians weigh potential benefits and risks in light of the patients' baseline status, comorbidities, and/or life expectancy. Therefore, we explored decision-making by physicians regarding EVT and/or thrombolysis in the setting of pre-stroke comorbidities/disability.

\section{Methods}

We conducted an international cross-sectional web-based survey (UNMASK-EVT) ${ }^{18}$ of stroke physicians to understand their current treatment practice and EVT/thrombolysis decisionmaking in acute stroke. Participants were assigned to 10 case scenarios from a pool of 22 and were asked how they would treat the patient in the given scenario, under their current practice conditions and under ideal conditions (i.e. assuming the absence of any local resource limitations or other practice constraints). Case scenarios were developed by consensus of the steering committee and were designed to represent common clinical situations. Each scenario was written to represent either a clear standard of care indication (guideline based) for treatment or a deviation from that standard of care, so that we could specifically assess how respondents interpreted the indications for treatment. The survey was distributed in waves by country/region leads in each region of the world to each region's available distribution list. Responses were obtained from November 26, 2017 to March 27, 2018. Approval was obtained from the local research ethics board, and participants provided electronic informed consent.

In total, 1330 stroke physicians (neurologists, interventional neuroradiologists, neurosurgeons, internists, geriatricians, and other physicians [including emergency] directly involved in acute stroke care) from 38 countries were invited to participate. Whereas geriatricians and internists may not play a role in acute stroke decision-making in North American centers, they often serve as acute stroke physicians in countries like the United Kingdom; consequently, some stroke physicians in our study from such practice settings were geriatricians and internists. ${ }^{19}$ No restrictions with respect to case volume or experience levels were applied, and participants had both academic and non-academic backgrounds.

Participants were simply asked to provide their favored treatment approach to 10 randomly assigned case scenarios, five involving major comorbidities (A: Stage-IV metastatic prostate cancer, B: heart failure, Chronic Obstructive Pulmonary Disease (COPD), and renal insufficiency requiring dialysis, $\mathrm{C}$ : nonmetastatic prostate cancer with anti-hormonal treatment, D: non-disabling (mild) cognitive impairment, and E: rheumatoid arthritis (RA) requiring care in a nursing home but without cognitive impairment). All five patients had proximal Internal Carotid Artery (ICA)/Middle Cerebral Artery (MCA) occlusions and were otherwise eligible for treatment with both alteplase and EVT. Of the other 17 scenarios, six were designed to represent "level-1A" evidence for which treatment with EVT and alteplase was clearly indicated per current AHA/ASA guidelines (see supplementary appendix). ${ }^{20}$

\section{Statistical Analysis}

The proportions of respondents who decided to proceed with EVT were determined and compared across scenarios to determine if presence of comorbidities would deter the physicians from selecting EVT. We first compared the proportion of 
respondents favoring EVT for the five comorbidity-related scenarios (examining the scenarios together and individually) to that for the six $1 \mathrm{~A}$ scenarios and then to that for all the remaining 17 scenarios.

We used logistic regression to determine the influence of each comorbidity-related factor (cancer [none, managed with hormonal therapy, vs metastatic], multiple comorbidities, non-disabling cognitive impairment, and dependent functional status with preserved cognition) on EVT decision rates under current resources and under assumed ideal conditions, unadjusted and adjusted for other scenario characteristics: baseline Alberta Stroke Program Early CT Score (ASPECTS), ${ }^{21}$ onset-to-presentation time, patient age, sex, National Institutes of Health Stroke Scale score, and occlusion site (ICA, M1, proximal or distal M2). We first did this analysis including the responses for only one comorbidity-related scenario at a time (examining the influence of each individual premorbid illness/disability factor) and then all the five scenarios (adjusting for all comorbidity-related factors), together with the "ideal" 1 A scenarios alone. We then repeated this process including the comorbidity-related and all 17 non-comorbidity-related scenarios, to see if the influence of comorbidity-related factors was different on accounting for all the other potentially "non-ideal" factors that may be encountered in practice. We used logistic regression to determine the influence of respondent characteristics (age, sex, speciality, region, hospital type [teaching/non-teaching], EVT cases/year) in univariable and multi-variable models, on EVT decision for the five comorbidity-related scenarios examined together and individually. Since respondents may have answered more or less of the same types of questions owing to the random scenario assignment, we adjusted the covariance matrix of the logistic regressions to account for lack of independence of observation within-respondent (or within-respondent correlations).

Similar analyses were performed to examine the decision to provide alteplase, with/without EVT.

Statistical significance was defined as $p<0.05$. Analyses were performed in STATA 13.1. Figures were created using Microsoft Power BI and the Mapbox Visual Plugin.

\section{Results}

We received responses from 607 physicians from 38 countries in different specialties. They provided 6070 scenario-based responses, with 1379 for five comorbidity-related scenarios (Table 1). Patients in comorbidity-related scenarios were generally older. Respondents had a median age of 44 years (interquartile range 39-50, Table 1), $15.9 \%$ were women, and the main specialities were neurology $(53.7 \%)$, neurosurgery $(13.3 \%)$, interventional neuroradiology $(28.5 \%)$, and geriatrics or internal medicine $(1.2 \%)$.

Respondents favored EVT in 1097/1379 (79.6\%) responses for the comorbidity-related scenarios under current resources and in $1140(82.7 \%)$ under assumed ideal conditions; these rates were lower than those for six scenarios with level-1A evidence (for which, EVT and alteplase were clearly indicated per current guidelines): $1510 / 1657$ (91.1\%, odds ratio [OR]: $0.38,95 \%$ confidence interval [CI] $0.31-0.47)$ and 1575 (95.1\%, OR: $0.25,0.19-0.33)$, respectively. Proportions favoring intravenous alteplase did not differ between comorbidity-related and level-1A scenarios (under current resources: $75.0 \%$ vs $72.2 \%$, OR: 1.15 , 0.99-1.33; assuming ideal conditions: $72.2 \%$ vs $69.5 \%$, OR:
1.14, 0.99-1.31). Comparing each individual comorbidity-related scenario to level-1A scenarios, rates favoring EVT were lower for scenarios A (metastatic prostate cancer), C (non-metastatic prostate cancer), and D (mild cognitive impairment/MCI) under current resources and when assuming ideal conditions (Supplementary Table 1). Corresponding rates favoring alteplase (with/without EVT) were lower for scenario A (metastatic prostate cancer, Supplementary Table 2) but higher for C (non-metastatic cancer) and $\mathrm{E}$ (dependent in nursing home), the latter difference explained by more respondents choosing EVT without alteplase for some 1-A scenarios. When all comorbidityrelated factors were considered in adjusted logistic regressions including comorbidity-related and level-1A scenarios (Supplementary Table 3), D (MCI) was the only scenario with lower EVT odds. On similar analyses for alteplase decisions, all scenarios except D (MCI) were associated with lower alteplase odds (Supplementary Table 4); respondents more often chose alteplase alone with MCI. That being said, this alteplase model had a very wide range of ORs, warranting a cautious interpretation.

In contrast, response rates favoring EVT with comorbidityrelated scenarios were higher than those seen when considering all the remaining scenarios (Figure 1): $3489 / 4691$ (74.4\%, OR: $1.34,1.17-1.54)$ under current resources and $3653(77.9 \%$, OR: $1.36,1.17-1.57)$ assuming ideal conditions, corresponding numbers for alteplase were 2832 (60.4\%, OR: 1.97, 1.73-2.24) and 2722 (58.0\%, OR: 1.87, 1.66-2.12). Respondents favored treatment as or more frequently in each of the five comorbidityrelated scenarios versus all others, except D (MCI) for EVT (Supplementary Table 5) and A (metastatic cancer) for alteplase (Supplementary Table 6). When all comorbidity-related factors were considered in adjusted logistic regressions, MCI and dependent status were associated with higher odds of favoring EVT (Table 2) and alteplase (Supplementary Table 7), while the rest of the comorbidities (metastatic/non-metastatic cancer and multi-system dysfunction [B]) were associated with lower alteplase odds. Older age and female sex were associated with lower EVT odds, but while female sex was also associated with lower alteplase odds, older age was associated with higher odds.

On multivariable logistic regression examining EVT decisionmaking for the comorbidity-related scenarios, adjusted for respondent characteristics (Table 3), EVT odds were higher with more EVT cases/year reported per respondent and center, with practicing in East Asia versus North America, and lower with more alteplase cases/center/year. With assuming ideal conditions, practising in East Asia remained significant. Compared to respondents in East Asia, those in South Asia were less likely to choose EVT under current resources (adjusted odds ratio [aOR]: $0.33,0.16-0.67, p=0.002$, regional heterogeneity illustrated in Figure 2), but this difference was attenuated on assuming ideal conditions (aOR: 0.81, 0.35-1.85, $p=0.61$ ). On similar multivariable regression for alteplase decisions (Supplementary Table 8), alteplase odds were lower with interventional neuroradiologists vs neurologists (both under current resources and assuming ideal conditions), more EVT cases/center/year, and female versus male respondents (under current resources). On examining each individual scenario, EVT odds were higher under current resources for neuroradiologists and neurosurgeons versus neurologists in scenario A (metastatic cancer, e.g., aOR [neuroradiologist]: 3.31, 1.15-9.53), with more EVT cases/ respondent/year in scenarios $\mathrm{C}$ (non-metastatic cancer, aOR: 


\section{Table 1: Baseline scenario and respondent characteristics for the comorbidity-related case scenarios}

\begin{tabular}{|c|c|c|}
\hline Scenario characteristics & Comorbidity scenarios $(n=5)$ & All other scenarios $(n=17)$ \\
\hline Patient age - Median (IQR) & 80 years $(6)$ & 69 years $(17)$ \\
\hline Patient sex, Female $-n(\%)$ & $2(40.0 \%)$ & $8(47.1 \%)$ \\
\hline Baseline aspects - Median (IQR) & $7(1)$ & $7(2)$ \\
\hline Onset-to-presentation time - Median (IQR) & $3 \mathrm{~h}(1)$ & $3 \mathrm{~h}(1)$ \\
\hline \multicolumn{3}{|l|}{ Site of occlusion $-n(\%)$} \\
\hline ICA & $1(20.0 \%)$ & $4(23.5 \%)$ \\
\hline M1 & $3(60.0 \%)$ & $9(52.9 \%)$ \\
\hline Proximal M2 & $1(20.0 \%)$ & $3(17.6 \%)$ \\
\hline Distal M2 & & $1(5.9 \%)$ \\
\hline Dependent baseline status $-n(\%)$ & $1(20.0 \%)^{*}$ & \\
\hline Prostate cancer $-n(\%)$ & $2(40.0 \%), 1$ metastatic & \\
\hline Heart failure $-n(\%)$ & $1(20.0 \%)^{+}$ & \\
\hline COPD $-n(\%)$ & $1(20.0 \%)^{+}$ & \\
\hline Renal disease with dialysis $-n(\%)$ & $1(20.0 \%)^{+}$ & \\
\hline Mild cognitive impairment $-n(\%)$ & $1(20.0 \%)$ & \\
\hline Rheumatoid arthritis $-n(\%)$ & $1(20.0 \%)^{*}$ & \\
\hline Respondent characteristics & Comorbidity scenarios (1379 responses) & All other scenarios (4691) \\
\hline Respondent age - Median (IQR) & $44(11)$ & $44(11)$ \\
\hline Respondent sex, Female $-n(\%)$ & $242(17.6)$ & $728(15.5)$ \\
\hline \multicolumn{3}{|l|}{ Speciality $-n(\%)$} \\
\hline Neurologist & $762(55.3)$ & $2498(53.3)$ \\
\hline Interventional neuroradiologist & $379(27.5)$ & $1351(28.8)$ \\
\hline Neurosurgeon & $177(12.8)$ & $633(13.5)$ \\
\hline Geriatrician/internist & $15(1.1)$ & $55(1.2)$ \\
\hline Other & $46(3.3)$ & $154(3.3)$ \\
\hline Years of experience - Median (IQR) & $13(12)$ & $13(12)$ \\
\hline \multicolumn{3}{|l|}{ Region $-n(\%)$} \\
\hline North America & $501(36.3)$ & $1679(35.8)$ \\
\hline Australia/New Zealand & $86(6.2)$ & $294(6.3)$ \\
\hline East Asia & $266(19.3)$ & $934(19.9)$ \\
\hline Europe & $299(21.7)$ & $1061(22.6)$ \\
\hline Middle-East & $34(2.5)$ & $106(2.3)$ \\
\hline South America & $100(7.3)$ & $310(6.6)$ \\
\hline South Asia & $93(6.7)$ & $307(6.5)$ \\
\hline \multicolumn{3}{|l|}{ Hospital type $-n(\%)$} \\
\hline Teaching hospital & $1251(90.7)$ & $4259(90.8)$ \\
\hline Non-teaching & $128(9.3)$ & $432(9.2)$ \\
\hline Strokes/respondent/year - Median (IQR) & $100(250)$ & $100(200)$ \\
\hline EVT/respondent/year - Median (IQR) & $30(35)$ & $30(35)$ \\
\hline Alteplase cases/center/year - Median (IQR) & $100(120)$ & $100(120)$ \\
\hline EVT cases/center/year - Median (IQR) & $70(90)$ & $65(90)$ \\
\hline
\end{tabular}

Items tagged with a $*$ or ${ }^{+}$were represented in the same scenario (each symbol being one scenario).

$1.03,1.003-1.05)$ and D (MCI), and with practising in East Asia in scenario D (MCI, aOR vs North America: 3.51, 1.41-8.75). Although respondents in Australia/New Zealand also seemed to favor EVT more than those in other parts of the world $(78.6 \%$ vs $60 \%$ in North America, Figure 2), higher EVT odds were not seen for this region on multivariable analysis. EVT odds were lower 

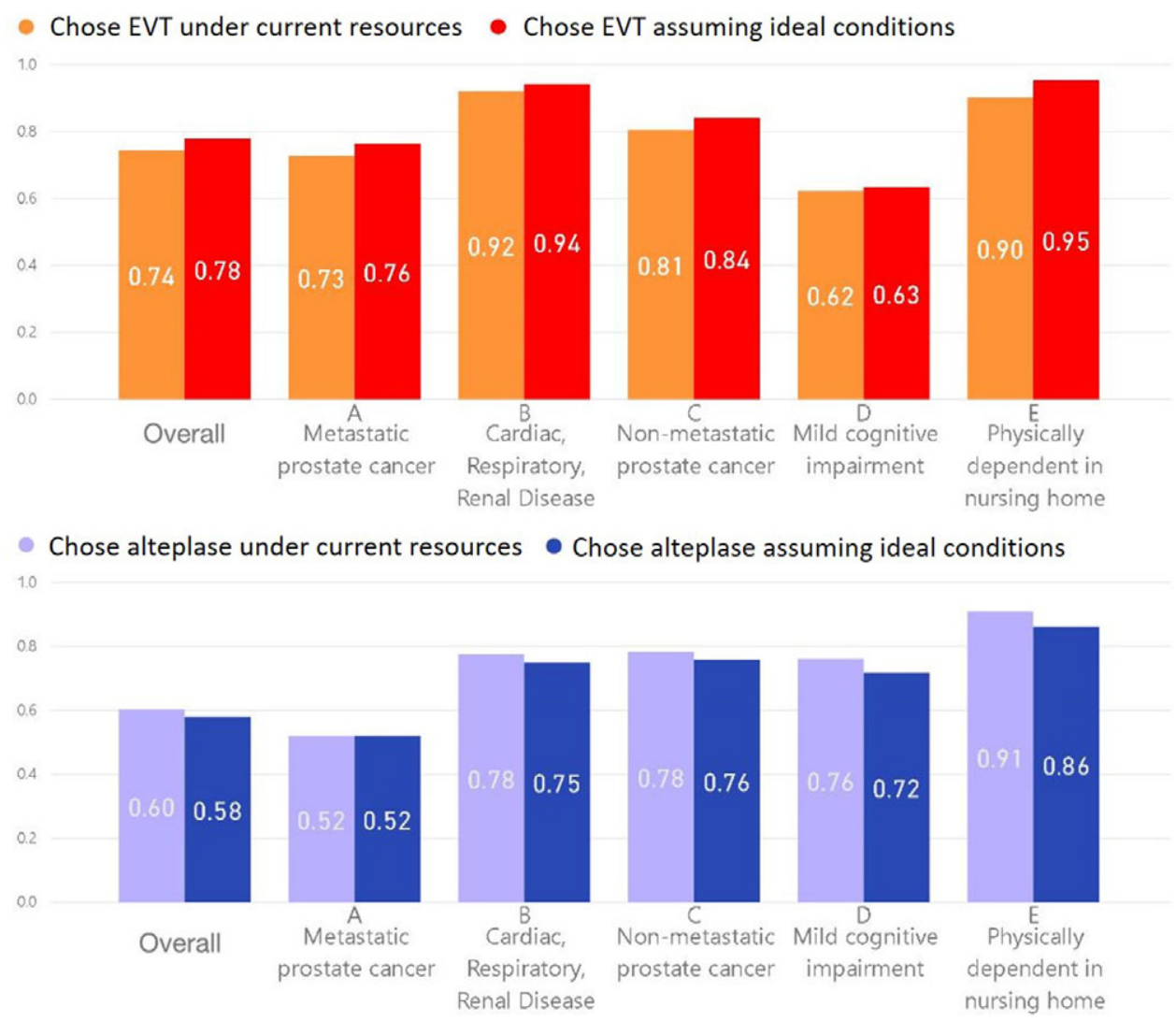

Figure 1: The proportion of respondents who chose EVT and alteplase under current resources (i.e. assuming their current practice conditions) and when assuming ideal conditions (i.e. assuming the absence of any local resource limitations or other practice constraints) for all scenarios (overall) and for each of the five comorbidity-related scenarios.

under current resources with more alteplase cases/center/year in scenarios A (metastatic prostate cancer) and B (heart failure/ COPD/dialysis, aOR: 0.995, 0.992-0.999), and with geriatricians/internists versus neurologists (aOR: 0.04, 0.005-0.35) or practicing in Australia/New Zealand in scenario E (nursing home with RA; aOR vs North America: 0.08, 0.02-0.40). Alteplase odds were lower with more EVT cases/center/year in scenario A (metastatic cancer, aOR [under current resources]: 0.993, 0.986-0.999), with interventional neuroradiologists in scenario B (heart failure/COPD/dialysis, aOR vs neurologists: 0.30 , 0.11-0.82), with geriatricians/internists in scenario $\mathrm{E}$ (nursing home with RA; aOR [assuming ideal conditions]: 0.05, 0.0040.61 ), and with practicing in Europe versus North America in scenarios A (metastatic cancer, aOR [assuming ideal conditions]: $0.34,0.15-0.79$ ) and B (heart failure/COPD/dialysis).

\section{Discussion}

In this international multidisciplinary scenario-based survey, respondents were less likely to favor EVT in the presence of patient comorbidities - particularly cognitive impairment compared to ideal scenarios meeting level-1A evidence, but by a small absolute difference. However, when considering all scenarios presented, the presence of comorbidities and disability did not deter respondents from pursuing EVT, especially on accounting for additional factors like baseline stroke severity, extent of ischemic changes (ASPECTS), and occlusion site. On the other hand, respondents were less likely to include alteplase in the setting of metastatic/non-metastatic cancer or multisystem dysfunction but were comfortable giving alteplase with pre-stroke dependence or MCI. These findings have implications for our understanding of physician decision-making in acute stroke and for informing the care of patients with comorbidities.

First, the finding that respondents less often favored EVT/ alteplase in the setting of comorbidity when compared to ideal level-1A scenarios unsurprisingly confirms the relative uncertainty experienced by physicians when faced with premorbid illness/disability. However, on adjusting for other scenario characteristics, cognitive impairment was the only comorbidityrelated factor associated with lower EVT odds versus level-1A scenarios. This suggests that cognitive impairment is overvalued by stroke physicians in EVT decision-making, especially since we specified the patient had MCI or non-disabling cognitive impairment (versus dementia). While there are few data on thrombectomy in these patients, observational studies of thrombolysis in patients with dementia suggest that mortality and hemorrhage risks are comparable to those without dementia, though functional outcomes may be worse. ${ }^{22-24}$ In this regard, it is noteworthy that most respondents chose alteplase in the scenario with MCI.

Second, the absence of any consistent association between the comorbidities presented and decreased odds of favoring EVT (on considering all scenarios) demonstrate a lack of consensus among 


\section{Table 2: Multivariable logistic regression for the association of comorbidity-related factors (bolded) with the decision to pursue EVT under current resources and under assumed ideal conditions, adjusted for key scenario characteristics, containing all comorbidity-related and non-comorbidity-related scenarios $\left(5792\right.$ responses, $\left.P>\chi^{2}<0.0001\right)$}

\begin{tabular}{|c|c|c|}
\hline & Under current resources & Assuming ideal conditions \\
\hline Factor & aOR $(95 \% \mathrm{CI})$ & $\mathrm{aOR}(95 \% \mathrm{CI})$ \\
\hline \multicolumn{3}{|l|}{ Cancer } \\
\hline No cancer & Reference & Reference \\
\hline Non-metastatic & $0.99(0.70-1.40)$ & $0.81(0.55-1.21)$ \\
\hline Metastatic prostate.cancer & $1.02(0.72-1.44)$ & $0.99(0.66-1.49)$ \\
\hline Heart failure, COPD, renal disease (dialysis) & $0.99(0.65-1.50)$ & $0.77(0.45-1.31)$ \\
\hline Mild cognitive impairment & $1.76(1.29-2.41)$ & $1.74(1.25-2.42)$ \\
\hline Dependent in nursing-home from RA (normal cognition) & $2.85(1.91-4.25)$ & $4.37(2.48-7.69)$ \\
\hline ASPECTS (per 1-point increase) & $1.47(1.39-1.55)$ & $1.66(1.55-1.78)$ \\
\hline NIHSS (per 1-point increase) & $1.14(1.12-1.16)$ & $1.18(1.15-1.21)$ \\
\hline Onset-to-presentation time (per 1-hour increase) & $0.83(0.79-0.88)$ & $0.80(0.76-0.85)$ \\
\hline Age (per 1-year increase) & $0.99(0.98-0.99)$ & $0.98(0.98-0.99)$ \\
\hline Sex - Female & $0.68(0.55-0.84)$ & $0.66(0.49-0.88)$ \\
\hline \multicolumn{3}{|l|}{ Occlusion location } \\
\hline ICA & Reference & Reference \\
\hline M1 & $2.30(1.77-2.98)$ & $2.68(1.92-3.74)$ \\
\hline Proximal M2 & $2.02(1.43-2.86)$ & $2.44(1.61-3.68)$ \\
\hline Distal M2 & $0.11(0.08-0.15)$ & $0.06(0.04-0.09)$ \\
\hline
\end{tabular}

stroke experts about excluding patients based on pre-stroke comorbidity/disability. There is no mechanistic basis for why patients with comorbidities cannot benefit from EVT/alteplase, although the degree of benefit may not be as robust as in healthier patients. We know that these patients accumulate disability and experience worse clinical and health economic outcomes if acute treatments are routinely withheld, ${ }^{25}$ and there is preliminary evidence that some of these patients can retain their premorbid state with EVT/alteplase without increased risk of harm. ${ }^{26}$ Adding in the absence of any clear comorbidity-based rationale for decision-making among our surveyed experts, the routine exclusion of patients based on some combination of pre-stroke comorbidities/disability may not be tenable. A crucial modifier in practice will be the values of patients as voiced by themselves or alternative decision-makers. The perspectives of patients with pre-stroke multi-morbidity/disability regarding EVT/alteplase remain an avenue for further study.

Third, our results demonstrate uncertainty among respondents regarding the added value of intravenous alteplase when pursuing EVT in setting of comorbidities. We found alteplase was often favored in scenarios where respondents were reluctant to pursue EVT (older age, MCI) but was often left out in other comorbiditybased scenarios where respondents chose EVT, despite no obvious alteplase contraindication. Respondents reporting more EVT cases/year and interventional neuroradiologists were more likely to forego alteplase in scenarios where they favored EVT, suggesting a perceived lack of benefit or risk of harm. This equipoise may indicate the value of trials comparing alteplase plus EVT to EVT alone, ${ }^{27,28}$ but unless such studies enroll patients with pre-stroke comorbidity/disability, they are unlikely to definitively settle this issue.

Fourth, our findings that patients' older age and female sex were associated with lower odds of respondents favoring EVT (and alteplase for female sex), whereas comorbidities themselves were not, raise questions about implicit biases in EVT decisionmaking. Age and sex may well have contributed to the apparent instability of the association of certain comorbidities with lower enthusiasm for treatment in our analysis. For example, while the MCI scenario had an overall lower proportion of respondents choosing EVT, the MCI characteristic itself was associated with higher rather than lower odds once adjusting for other scenario characteristics including age and sex (the patient in this scenario was an 85-year-old woman). In individual patient meta-analyses, age and sex did not modify EVT treatment effect. ${ }^{1}$ Agism has been noted in interventional stroke studies, ${ }^{29}$ and older patients and women appear less likely to receive appropriate acute stroke care. $^{30,31}$ Our finding that age and sex further modify EVT decisions merits further validation.

Fifth, our results imply that EVT/alteplase decision-making in the setting of comorbidities is strongly associated with physician characteristics. Interventional neuroradiologists and neurosurgeons more often favored EVT than neurologists, as did those reporting more EVT cases/year, whereas geriatricians/internists were less likely to choose EVT/alteplase, particularly for an already dependent patient. Regional differences included respondents in East Asia more often favoring EVT than North-American counterparts, whereas those in South Asia less often chose EVT under current resources but became more likely to do so on 
Table 3: Multivariable logistic regression for the association of respondent characteristics with the decision to pursue EVT for the five comorbidity-related scenarios under current resources and under assumed ideal conditions (1379 responses, $P>\chi^{2}<$ 0.0001)

\begin{tabular}{|c|c|c|}
\hline & Under current resources & Assuming ideal conditions \\
\hline Factor & aOR $(95 \%$ CI) & aOR $(95 \% \mathrm{CI})$ \\
\hline Age & $1.00(0.96-1.05)$ & $1.01(0.97-1.06)$ \\
\hline \multicolumn{3}{|l|}{ Sex } \\
\hline Male & Reference & Reference \\
\hline Not declared & $1.57(0.14-17.5)$ & All in favor $(n=6)$ \\
\hline Female & $0.92(0.63-1.34)$ & $0.80(0.55-1.17)$ \\
\hline \multicolumn{3}{|l|}{ Speciality } \\
\hline Neurologist & Reference & Reference \\
\hline Interventional neuroradiologist & $1.30(0.81-2.09)$ & $1.01(0.63-1.61)$ \\
\hline Neurosurgeon & $1.43(0.80-2.58)$ & $0.94(0.54-1.62)$ \\
\hline Geriatrician/internist & $0.39(0.15-1.04)$ & $0.82(0.28-2.41)$ \\
\hline Other & $0.84(0.44-1.60)$ & $1.48(0.65-3.38)$ \\
\hline Years of experience & $1.01(0.96-1.05)$ & $0.97(0.93-1.02)$ \\
\hline \multicolumn{3}{|l|}{ Region } \\
\hline North America & Reference & Reference \\
\hline Australia/New Zealand & $0.81(0.48-1.36)$ & $1.17(0.63-2.20)$ \\
\hline East Asia & $2.27(1.30-3.98)$ & $2.54(1.51-4.28)$ \\
\hline Europe & $0.75(0.48-1.18)$ & $1.11(0.73-1.70)$ \\
\hline Middle East & $1.99(0.73-5.41)$ & $1.10(0.43-2.81)$ \\
\hline South America & $1.00(0.51-1.96)$ & $1.37(0.72-2.59)$ \\
\hline South Asia & $0.74(0.39-1.40)$ & $2.05(0.97-4.32)$ \\
\hline \multicolumn{3}{|l|}{ Hospital type } \\
\hline Teaching hospital & Reference & Reference \\
\hline Non-teaching & $1.28(0.72-2.27)$ & $1.30(0.72-2.38)$ \\
\hline Strokes/respondent/year & $1.00(1.00-1.00)$ & $1.00(1.00-1.00)$ \\
\hline EVT/respondent/year & $1.01(1.0004-1.01)$ & $1.01(0.99-1.01)$ \\
\hline Alteplase cases/center/year & $0.999(0.997-0.999)$ & $1.00(1.00-1.00)$ \\
\hline EVT cases/center/year & $1.01(1.003-1.01)$ & $1.004(1.001-1.007)$ \\
\hline
\end{tabular}

assuming ideal conditions. This further emphasizes the importance of investing in stroke-care resources in Asia, where there is a known disparity between availability of acute stroke infrastructure in East Asia (high) versus the rest of the region. ${ }^{32}$

A few limitations merit discussion. While the overall completion rate was high $(45.6 \%)$ compared to other surveys, we only had a few participants from some countries. As there is no comprehensive international register of stroke physicians, participant enrollment relied on institutional networks and collaborations, potentially limiting the study's representativeness. In addition, survey-based data may not accurately reflect decision-making in routine practice, although care was taken to frame the scenarios to realistically reflect routine practice. However, the physicians' real-life decisions may still differ from the hypothetical ones made in these case scenarios. Our ability to assess the relative importance of each comorbidity was limited by respondents only being presented with one comorbidity factor (e.g. cancer or MCI) at a time. Future studies of the influence of comorbidities on EVT/alteplase decisions may consider presenting respondents with multiple different combinations of these comorbidity factors. Since this study ultimately relied on mock scenarios rather than real-life cases, there were expected limitations in the ranges, permutations, and combinations of demographic and clinical variables that could be captured within those cases, which may have contributed to instability or wide ranges of ORs in some of the multi-variable logistic models. Although the scenarios were chosen by consensus among a steering committee of stroke experts, the range or severity of the comorbidities presented may not reflect the experiences of stroke physicians around the world. In addition, we were unable to calculate a response rate for our survey; the denominator for distribution is not known because each list was known only to each country/ region lead and distribution occurred in waves, with the surveys likely forwarded to known colleagues and thereby resulting in 


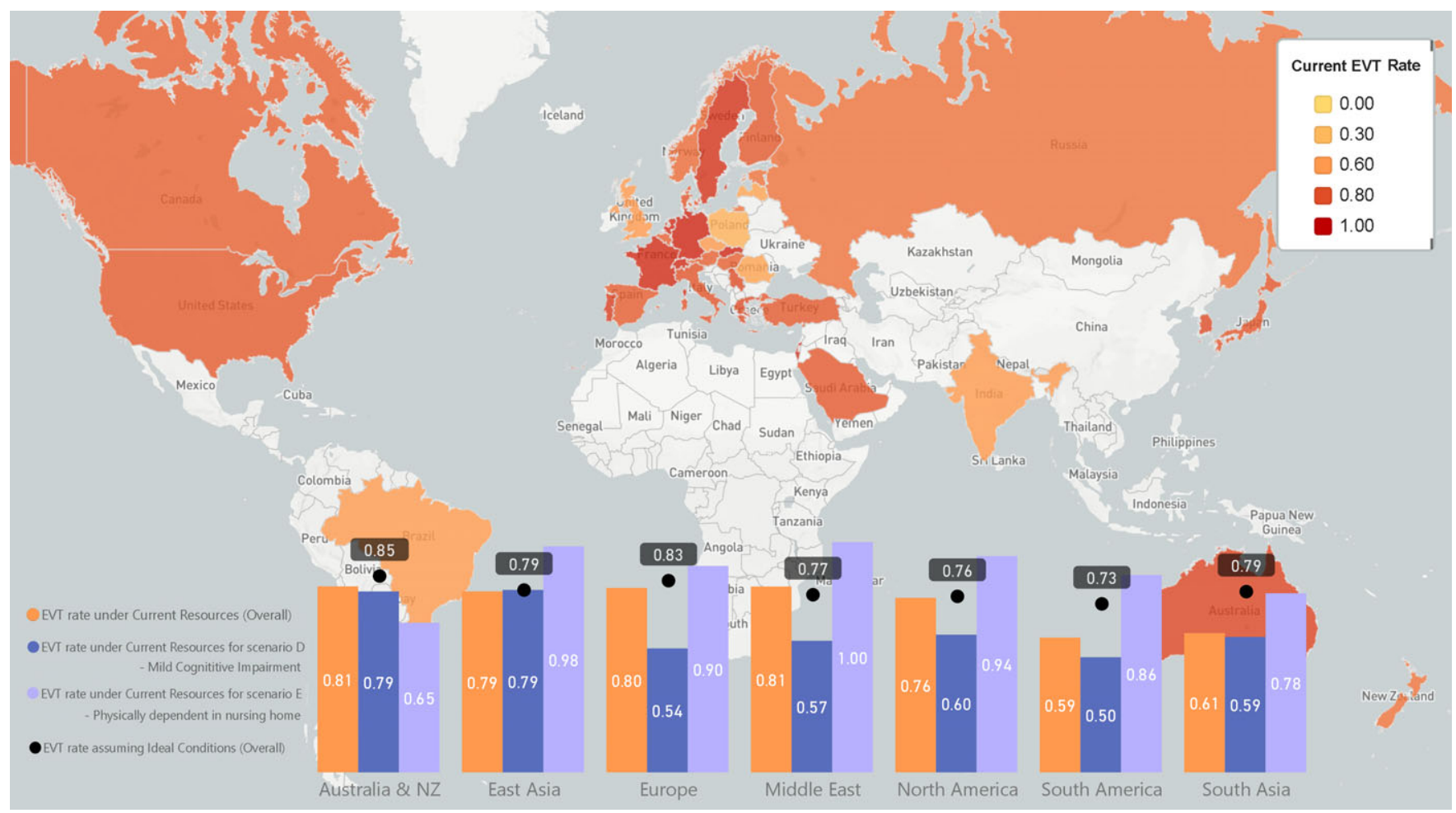

Figure 2: The proportion of respondents from key world regions who chose EVT under current resources for all scenarios (overall) and for comorbidity-related scenarios $D$ (mild cognitive impairment) and $E$ (physically dependent in nursing home). The proportion of respondents who chose EVT assuming ideal conditions (overall) in each region is also shown. The world map shows the overall proportion of responses in each country that favored EVT under current resources, when considering all 22 scenarios. The color scheme is based on a spectrum for the proportion ranging from 0.00 to 1.00, with key colors and corresponding proportions indicated in the legend on the upper right. Hues of yellow evolving to orange represent the range of proportions from 0.00 to 0.60 , with the hues then evolving from orange to red as the proportion rises from 0.60 to 1.00 . Countries that were either not captured in the study or for which we had fewer than three respondents appear white.

challenges to the representativeness of our sample. Therefore, our results must be interpreted knowing the limitations of this type of survey-based approach to assessing attitudes about standards of care. Despite these limitations, our findings can help further understand the influence of comorbidities and premorbid disability on treatment attitudes of stroke physicians across a broad, international, multidisciplinary spectrum.

\section{Conclusion}

In this international survey, we sought to explore EVT/ alteplase decision-making by stroke experts in the setting of comorbidity/disability. Moderate and even severe disabling comorbidities did not consistently deter experts from choosing EVT in this international survey, arguing against the routine exclusion of such patients. However, intravenous alteplase was often foregone in these scenarios when respondents chose EVT. Differences in approach based on patient age and sex merit further investigation. Inter-speciality and regional differences in EVT/alteplase decision-making could potentially be mitigated by improving access to stroke care resources.

\section{Disclosures}

Aravind Ganesh reports membership in the editorial boards of Neurology, Neurology: Clinical Practice, and Stroke; speaker honoraria from NHS Health Education England; consulting fees from MD Analytics, MyMedicalPanel, Adkins Research Group, and Genome BC; research support from The Rhodes Trust, Wellcome Trust, the University of Calgary, Alberta Innovates, the Canadian Cardiovascular Society, and the Canadian Institutes of Health Information; stock/stock options from SnapDx, TheRounds.ca, and Advanced Health Analytics (AHA Health Ltd); and a provisional patent application (US 63/024,239) for a system to deliver remote ischemic conditioning or other cuff-based therapies. Gustavo Saposnik reports a Heart and Stroke Foundation of Canada Career Award. Mayank Goyal reports personal fees from Medtronic, personal fees from Stryker, personal fees from Microvention, personal fees from Mentice, outside the submitted work; in addition, Dr. Goyal has a patent Systems of Stroke Diagnosis licensed to GE Healthcare. Michael Hill reports grants from Covidien (Medtronic), personal fees from Merck, non-financial support from Hoffman La Roche Canada, outside the submitted work. In addition, Dr. Hill has a patent 62/086,077 for triaging systems in ischemic stroke issued and Research support from Alberta Innovates Health Solutions, Heart \& Stroke Foundation, Hotchkiss Brain Institute, Canadian Stroke Prevention Intervention Networks (Institute of Circulatory and Respiratory Health, CIHR), Calgary Stroke Program, Department of Clinical Neurosciences, University of Calgary; nonfinancial support from Alberta Health Services; stock in Calgary Scientific Incorporated, a company focusing on medical imaging software. Bijoy Menon reports personal fees from Penumbra Incorporated, outside the submitted work. In addition, Dr. Menon has a patent 62/086,077 for triaging systems in ischemic stroke issued and 
Membership of the Steering/Executive Committee, ESCAPE trial with support from Covidien; Site Principal Investigator, SOCRATES Trial, sponsored by Astra Zeneca; research funding from Canadian Institutes of Health Research (CIHR), Heart and Stroke Foundation of Canada, Alberta Innovates Health Solutions, Hotchkiss Brain Institute and Faculty of Medicine, University of Calgary and salary support from the CIHR New Investigator Award and Heart and Stroke Foundation/University of Calgary Professorship in Stroke Imaging. Johanna Ospel reports funding support from the Julia Bangerter Rhyner Foundation, University of Basel Research Foundation, and Freiwillige Akademische Gesellschaft Basel. The remaining authors have nothing to disclose.

\section{ACKNOWLEDGEMENTS}

The authors are most grateful to all the physicians who participated in the study.

\section{STATEMENT OF AUTHORShIP}

AG performed statistical analysis and interpretation, wrote and revised the manuscript. NK and JMO acquired data, performed statistical analysis, and revised the manuscript. ATW and MMF were involved in study concept and design, data acquisition, and manuscript revision. MG, MAA, MDH, BKM, and GS conceived and designed the study, provided supervision and funding, analyzed and interpreted data, and revised the manuscript.

\section{SUPPLEMENTARY MATERIAL}

To view supplementary material for this article, please visit https://doi.org/10.1017/cjn.2020.158.

\section{FUNDING}

The study was funded by Stryker Inc. through an unrestricted research grant to the University of Calgary. The company was not involved in the design, execution, analysis, interpretation, or reporting of the results.

\section{REFERENCES}

1. Goyal M, Menon BK, van Zwam WH, et al. Endovascular thrombectomy after large-vessel ischaemic stroke: a meta-analysis of individual patient data from five randomised trials. The Lancet. 2016;387(10029):1723-31.

2. Nogueira RG, Jadhav AP, Haussen DC, et al. Thrombectomy 6 to 24 hours after stroke with a mismatch between deficit and infarct. $\mathrm{N}$ Engl J Med. 2018;378(1):11-21.

3. Albers GW, Marks MP, Kemp S, et al. Thrombectomy for stroke at 6 to 16 hours with selection by perfusion imaging. N Engl J Med. 2018;378(8):708-18.

4. Emberson J, Lees KR, Lyden P, et al. Effect of treatment delay, age, and stroke severity on the effects of intravenous thrombolysis with alteplase for acute ischaemic stroke: a meta-analysis of individual patient data from randomised trials. Lancet 2014; 384(9958):1929-35.

5. Ma H, Campbell BCV, Parsons MW, et al. Thrombolysis guided by perfusion imaging up to 9 hours after onset of stroke. N Engl J Med. 2019;380(19):1795-803.

6. Thomalla G, Simonsen CZ, Boutitie F, et al. MRI-guided thrombolysis for stroke with unknown time of onset. N Engl J Med. 2018;379(7):611-22.
7. Ganesh A, Goyal M. Thrombectomy for acute ischemic stroke: recent insights and future directions. Curr Neurol Neurosci Rep. 2018;18(9):59.

8. Quinn TJ, Taylor-Rowan M, Coyte A, et al. Pre-stroke modified Rankin scale: evaluation of validity, prognostic accuracy, and association with treatment. Front Neurol. 2017;8:275.

9. Ganesh A, Luengo-Fernandez R, Wharton RM, Rothwell PM, Oxford Vascular S. Ordinal vs dichotomous analyses of modified Rankin scale, 5-year outcome, and cost of stroke. Neurology 2018;91(21):e1951-e60.

10. Cappellari M, Bosco M, Forlivesi S, et al. Reasons for exclusion from intravenous thrombolysis in stroke patients admitted to the stroke unit. J Thromb Thrombolysis 2016;42(4):593-9.

11. Kwok CS, Clark A, Ford GA, et al. Association between prestroke disability and inpatient mortality and length of acute hospital stay after acute stroke. J Am Geriatr Soc. 2012;60(4):726-32.

12. Schmidt M, Jacobsen JB, Johnsen SP, Botker HE, Sorensen HT. Eighteen-year trends in stroke mortality and the prognostic influence of comorbidity. Neurology 2014;82(4):340-50.

13. Borggrefe J, Gluck B, Maus V, et al. Clinical outcome after mechanical thrombectomy in patients with diabetes with major ischemic stroke of the anterior circulation. World Neurosurg. 2018;120:e212-e20.

14. Kurre W, Aguilar-Perez M, Niehaus L, et al. Predictors of outcome after mechanical thrombectomy for anterior circulation large vessel occlusion in patients aged $\geq 80$ years. Cerebrovasc Dis 2013;36(5-6):430-6.

15. Kamel H, Patel N, Rao VA, et al. The totaled health risks in vascular events (THRIVE) score predicts ischemic stroke outcomes independent of thrombolytic therapy in the NINDS tPA trial. J Stroke Cerebrovascular Dis 2013;22(7):1111-6.

16. Lu GD, Ren ZQ, Zhang JX, Zu QQ, Shi HB. Effects of diabetes mellitus and admission glucose in patients receiving mechanical thrombectomy: a systematic review and meta-analysis. Neurocritical Care 2018;29(3):426-34.

17. Gallacher KI, Jani BD, Hanlon P, Nicholl BI, Mair FS. Multimorbidity in stroke. Stroke 2019;50(7):1919-26.

18. Saposnik G, Menon BK, Kashani N, et al. Factors associated with the decision-making on endovascular thrombectomy for the management of acute ischemic stroke. Stroke 2019;50(9):2441-2447.

19. Oliver D, Burns E. Geriatric medicine and geriatricians in the UK. How they relate to acute and general internal medicine and what the future might hold? Future Hosp J. 2016;3(1):49-54.

20. Powers WJ, Rabinstein AA, Ackerson T, et al. 2018 Guidelines for the early management of patients with acute ischemic stroke: a guideline for healthcare professionals from the American Heart Association/American Stroke Association. Stroke 2018;49(3): e46-e110.

21. Barber PA, Demchuk AM, Zhang J, Buchan AM. Validity and reliability of a quantitative computed tomography score in predicting outcome of hyperacute stroke before thrombolytic therapy. ASPECTS Study Group. Alberta Stroke Programme Early CT Score. Lancet 2000;355(9216):1670-4.

22. Saposnik G, Kapral MK, Cote R, et al. Is pre-existing dementia an independent predictor of outcome after stroke? A propensity score-matched analysis. J Neurol. 2012;259(11):2366-75.

23. Alshekhlee A, Li CC, Chuang SY, et al. Does dementia increase risk of thrombolysis? A case-control study. Neurology 2011;76(18): $1575-80$.

24. Zupanic E, von Euler M, Kareholt I, et al. Thrombolysis in acute ischemic stroke in patients with dementia: a Swedish registry study. Neurology 2017;89(18):1860-8.

25. Ganesh A, Luengo-Fernandez R, Pendlebury ST, Rothwell PM. Long-term consequences of worsened poststroke status in patients with premorbid disability. Stroke 2018;49(10):2430-6.

26. Goldhoorn RB, Verhagen M, Dippel DWJ, et al. Safety and outcome of endovascular treatment in prestroke-dependent patients. Stroke 2018;49(10):2406-14.

27. SWIFT DIRECT. The SWIFT DIRECT trial; 2018. Available at: https://www.swift-direct.ch/; accessed June 11, 2020.

28. Bellwald S, Weber R, Dobrocky $\mathrm{T}$, et al. Direct mechanical intervention versus bridging therapy in stroke patients eligible 
for intravenous thrombolysis: a pooled analysis of 2 registries. Stroke 2017;48(12):3282-8.

29. Hadbavna A, O'Neill D. Ageism in interventional stroke studies. J Am Geriatr Soc. 2013;61(11):2054-5.

30. Asdaghi N, Romano JG, Wang K, et al. Sex disparities in ischemic stroke care: FL-PR CReSD study (Florida-Puerto Rico collaboration to reduce stroke disparities). Stroke 2016;47(10):2618-26.
31. Kimball MM, Neal D, Waters MF, Hoh BL. Race and income disparity in ischemic stroke care: nationwide inpatient sample database, 2002 to 2008. J Stroke Cerebrovasc Dis. 2014;23(1): $17-24$.

32. Toyoda K, Koga M, Hayakawa M, Yamagami H. Acute reperfusion therapy and stroke care in Asia after successful endovascular trials. Stroke 2015;46(6):1474-81. 